\title{
Auditory Brain Stem Responses in the C57BL/6J Fragile X Syndrome-Knockout Mouse Model
}

\author{
Amita Chawla and Elizabeth A. McCullagh* \\ Department of Integrative Biology, Oklahoma State University, Stillwater, OK, United States
}

Sensory hypersensitivity, especially in the auditory system, is a common symptom in Fragile $X$ syndrome (FXS), the most common monogenic form of intellectual disability. However, linking phenotypes across genetic background strains of mouse models has been a challenge and could underly some of the issues with translatability of drug studies to the human condition. This study is the first to characterize the auditory brain stem response (ABR), a minimally invasive physiological readout of early auditory processing that is also used in humans, in a commonly used mouse background strain model of FXS, C57BL/6J. We measured morphological features of pinna and head and used $\mathrm{ABR}$ to measure the hearing range, and monaural and binaural auditory responses in hemizygous males, homozygous females, and heterozygous females compared with those in wild-type mice. Consistent with previous study, we showed no difference in morphological parameters across genotypes or sexes. There was no significant difference in hearing range between the sexes or genotypes, however there was a trend towards high frequency hearing loss in male FXS mice. In contrast, female mice

OPEN ACCESS

Edited by:

Randy J. Kulesza,

Lake Erie College of Osteopathic Medicine, United States

Reviewed by: Charles H. Large, Autifony Therapeutics Ltd. United Kingdom

Adrian Rodriguez-Contreras, City College of New York (CUNY), United States

*Correspondence:

Elizabeth A. McCullagh elizabeth.mccullagh@okstate.edu

Received: 28 October 2021 Accepted: 14 December 2021 Published: 17 January 2022

Citation:

Chawla A and McCullagh EA (2022) Auditory Brain Stem Responses in the C57BL/6J Fragile $X$ Syndrome-Knockout Mouse Model. Front. Integr. Neurosci. 15:803483. doi: 10.3389/fnint.2021.803483 with homozygous FXS had a decreased amplitude of wave IV of the monaural ABR, while there was no difference in males for amplitudes and no change in latency of ABR waveforms across sexes and genotypes. Finally, males with FXS had an increased latency of the binaural interaction component $(\mathrm{BIC})$ at 0 interaural timing difference compared with that in wild-type males. These findings further clarify auditory brain stem processing in FXS by adding more information across genetic background strains allowing for a better understanding of shared phenotypes.

Keywords: auditory brainstem response (ABR), Fragile X Syndrome, binaural hearing, sex differences, mouse model

\section{INTRODUCTION}

Fragile X syndrome (FXS) is the most common monogenic form of autism spectrum disorder (ASD) and shares many attributes of ASDs, including auditory hypersensitivity and other sensory disruptions (Abbeduto and Hagerman, 1997; Chen and Toth, 2001; Hagerman and Hagerman, 2002; Arnett et al., 2014). FXS is a tractable genetic model for ASD with several commercially available models, including the rat and mouse (The Dutch-Belgian Fragile X Consorthium et al., 1994; Till et al., 2015; Tian et al., 2017). Despite the common use of these models to study the FXS, phenotypes are not always shared between species and background strains, particularly for sensory processing. As a result, drug therapies have struggled to rescue the human disorder (Dahlhaus, 2018). One of the most common symptoms described in people with FXS and autism spectrum disorder (ASD) is auditory hypersensitivity (Ethridge et al., 2017; Stefanelli et al., 2020). 
Clinically, auditory phenotypes present as reduced auditory attention, impaired habituation to auditory stimuli, reduced prepulse inhibition of acoustic startle, and overall hypersensitivity to auditory conditions (reviewed in Sinclair et al., 2017; Rais et al., 2018; Razak et al., 2021) that have likely both cortical and subcortical origins. Indeed, much of the research in this area has focused on cortical measures of auditory phenotypes, which receive inputs from lower auditory regions that may also be disrupted but less likely to be measured clinically. The mechanisms that underly auditory alterations are unknown, but likely involve the entirety of the ascending pathway from the periphery to the cortex (reviewed in McCullagh et al., 2020b). A complete characterization of auditory processing from the periphery to cortex across sexes, background strains, and models is needed to fully understand shared phenotypes and circuitry involved in this common symptom.

The auditory brain stem is one brain region in the ascending auditory pathway that has been shown to have anatomical, physiological, and behavioral alterations in mouse models with FXS (Brown et al., 2010; Beebe et al., 2014; Wang et al., 2014, 2015; Rotschafer et al., 2015; Garcia-Pino et al., 2017; McCullagh et al., 2017, 2020a; Rotschafer and Cramer, 2017; Curry et al., 2018; El-Hassar et al., 2019; Lu, 2019) that likely underly or contribute to the overall auditory phenotypes exhibited in both humans and animal models. Much like auditory hypersensitivity in humans, mice exhibit changes to the prepulse inhibition to the acoustic startle response, abnormal EEG activity, and, in the most extreme form, audiogenic seizures when presented with loud sounds (Chen and Toth, 2001; Lovelace et al., 2018, 2020; McCullagh et al., 2020a), making them a potentially suitable model for this sensory phenotype. The auditory brain stem is the first site of binaural processing of sound location in the brain using interaural timing and level differences (i.e., ITD and ILD, respectively) to compute sound source locations (Grothe et al., 2010). This brain area is also involved in separating spatial channels allowing for complex listening environments. Disruptions in this spatial separation and binaural processing could lead to auditory hypersensitivity due to the inability to separate sound sources (Bronkhorst, 2015). One measure of auditory brain stem physiology, and binaural hearing, that can be directly translated between animal models and humans is the auditory brain stem response (ABR) (Laumen et al., 2016).

The $A B R$ is a minimally invasive physiological measure that allows for a simultaneous assessment of sound processing across multiple brain stem nuclei, as each wave of the ABR directly corresponds to distinct areas of the ascending auditory brain stem pathway. These features make the ABR an attractive translational tool. Indeed, recent evidence suggests that $A B R$ measurements are an early indicator of auditory dysfunction in ASD (Santos et al., 2017). ABRs can also be used to assess binaural hearing, which is essential for sound localization and hearing in noisy environments and often impaired in ASD (Visser et al., 2013). Monoaural ABRs can be recorded by stimulating each ear separately, and binaural responses can be generated by stimulating both ears simultaneously. The sum of the two monaural (i.e., left and right) responses should equal the binaural (i.e., both ear) responses since the recruited neural activity from each ear should be double when stimulated simultaneously. However, this is not the case, there is a difference that arises when the summed monoaural responses are subtracted from the binaural response, called the binaural interaction component (BIC). The BIC is thought to be a direct measure of binaural processing ability in humans and animals that requires the precise balance of excitatory and inhibitory drive in brain stem sound localization circuits (Laumen et al., 2016).

In this study, we reported on the hearing ability, using the ABR and morphological craniofacial and pinna features, of the most common mouse model with FXS, C57BL/6J across the sexes and females heterozygous for the Fmrl mutation. We hypothesized that there may be sex differences in ABRs independent of the FXS genotype, but that in addition, FXS animals are likely to have alterations in peak amplitude or latency of ABRs and impaired high-frequency hearing compared with wild-type consistent with work in other mouse strains with FXS (Kim et al., 2013; Rotschafer et al., 2015; El-Hassar et al., 2019). Establishing core auditory phenotypes across the sexes and different mouse strains is key to creating a toolbox of techniques that may translate to human FXS both to validate the utility of animal models to human conditions but also add to potential measures for the efficacy of the drug or other treatment options.

\section{MATERIALS AND METHODS}

All experiments complied with all applicable laws, National Institutes of Health guidelines, and were approved by the Oklahoma State University IACUC.

\section{Animals}

Experiments were conducted in C57BL/6J (stock \#000664, B6) wild-type background, hemizygous male, homozygous male and female, or heterozygous female Fmr1 mutant mice (B6.129P2Fmr ${ }^{T M 1 C g r} / \mathrm{J}$ stock $\# 003025, \mathrm{Fmrl}$ or Fmrl het, respectively) obtained from the Jackson Laboratory and bred at Oklahoma State University (Bar Harbor, ME, United States) (The DutchBelgian Fragile X Consorthium et al., 1994). Animals were generated for these experiments from stocks by both mixed and single genotype mating allowing for the creation of heterozygotes and some littermate controls, as well as maintenance of breeding lines. There was no significant main effect of litter (i.e., mixed or single genotype) for any of the experiments. Sex was treated as a biological variable, and differences between the sexes, when present, are noted in the results. The numbers of animals for each experiment used are listed in the figure legends and range from 6-10 animals per sex and genotype. Animals ranged in age from 62-120 days (i.e., average ages per genotype $89 \pm 4$ days B6, $101 \pm 3$ days Fmr1, and $97 \pm 4$ days Fmr1 het).

\section{Morphological Measures}

Features of animal's head, pinna, and body mass (weight) were measured for each genotype using 6 Inch Stainless Steel Electronic Vernier Calipers (DIGI-Science Accumatic digital caliper Gyros Precision Tools Monsey, NY, United States) and an electronic scale. The distance between the two pinnae (i.e., 
interpinna distance), distance from the nose to the middle of the pinna (i.e., nose to pinna distance), and pinna width and length were measured (Figure 1A). The effective diameter was calculated as the square root of pinna length times pinna width (Anbuhl et al., 2017).

\section{Auditory Brain Stem Responses}

Auditory brain stem response recordings were performed using similar methods from previously published study (Benichoux et al., 2018; McCullagh et al., 2020a; New et al., 2021). Animals were anesthetized using two mixtures of ketamine-xylazine $60 \mathrm{mg} / \mathrm{kg}$ ketamine and $10 \mathrm{mg} / \mathrm{kg}$ xylazine for initial induction followed by maintenance doses of $25 \mathrm{mg} / \mathrm{kg}$ ketamine and $12 \mathrm{mg} / \mathrm{kg}$ xylazine. Once anesthesia was confirmed by lack of a toe-pinch reflex, animals were transferred to a small sound attenuating chamber (Noise Barriers Lake Forest, IL, United States), and the body temperature was maintained using a water-pump heating pad. Subdermal needle electrodes were placed under the skin between the ears (i.e., apex), directly behind the apex in the nape (i.e., reference), and in the back leg for ground. This montage has been shown to be particularly effective in generating the BIC (Levine, 1981; Laumen et al., 2016). Evoked potentials from subdermal needle electrodes were acquired and amplified using Tucker-Davis Technologies (TDT, Alachua, FL, United States) RA4LI head stage and a TDT RA16PA preamplifier. Further amplification was provided by a TDT Multi I/O processor RZ5 connected to a PC with custom Python software for data recording. Data
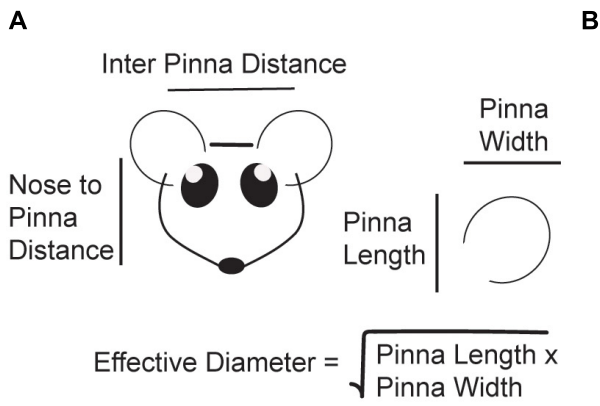

C

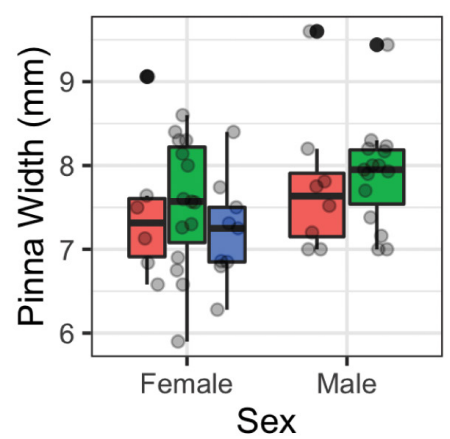

E

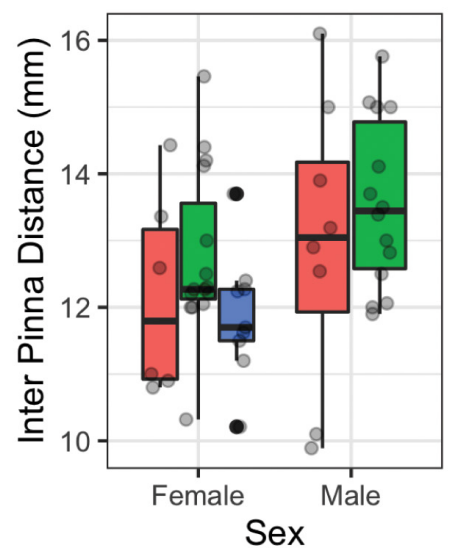

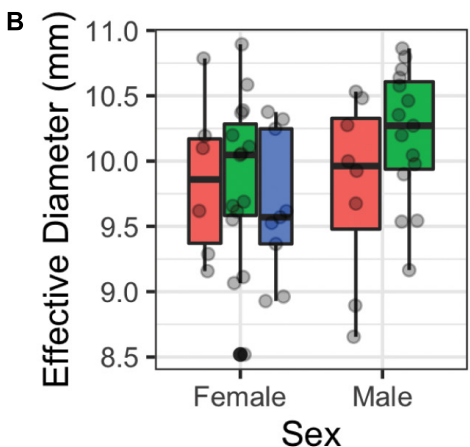

D

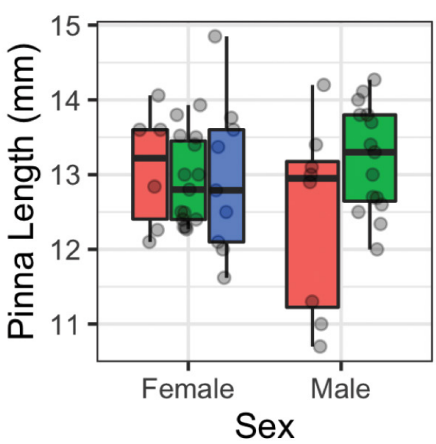

$\mathbf{F}$

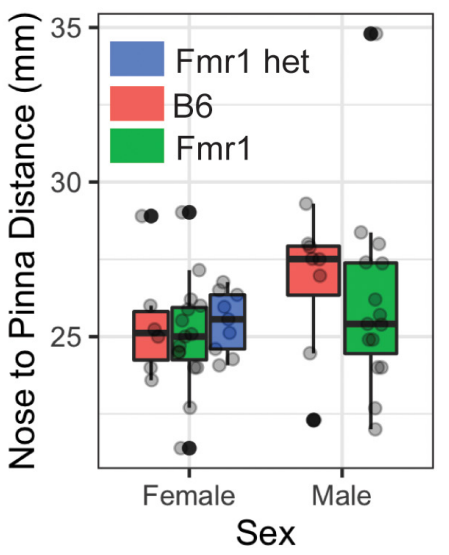

FIGURE 1 | Morphological features of Fragile X syndrome (FXS) mice. Pinna and head features (A) were measured between the sexes (X-axis) and genotypes (purple $=$ B6, teal $=$ Fmr1, and yellow $=$ Fmr1 het). There was no difference between the sexes or genotypes for any of the measures [effective diameter (B), pinna width (C), pinna length (D), interpinna length (E), or nose to pinna length (F)]. Data represent 6 B6, 15 Fmr1, and 9 Fmr1 het females and 8 B6 and 15 Fmr1 males. 
were averaged across 500-1,000 repetitions per condition and processed using a second-order $50-3,000 \mathrm{~Hz}$ filter over $12 \mathrm{~ms}$ of recording time.

Sound stimuli (refer below for varying types) were presented to the animal through TDT EC-1 electrostatic speakers (frequencies $32-46 \mathrm{kHz}$ ) or TDT MF-1 multifield speakers (frequencies 1-24 kHz and broadband clicks) coupled through custom earpieces fitted with Etymotic ER-7C probe microphones (Etymotic Research Inc., Elk Grove Village, IL, United States) for the in-ear calibration (Beutelmann et al., 2015). Sounds were generated using a TDT RP2.1 Real-Time processor controlled by the custom Python code at a sampling rate of $97656.25 \mathrm{~Hz}$. Sounds were presented at an interstimulus interval of $30 \mathrm{~ms}$ with a standard deviation of $5 \mathrm{~ms}$ (Laumen et al., 2016). An additional rejection threshold was set to eliminate high-amplitude heart rate responses from average traces and improve the signal-tonoise ratio.

\section{Audiogram}

The hearing range of animals was tested using the threshold for hearing across different frequencies (i.e., 1, 2, 4, 8, 16, 24, $32,46 \mathrm{kHz}$ ) of sound. Threshold was determined using a visual detection method (Brittan-Powell and Dooling, 2004), or the lowest level (dB SPL) a response could be detected. Audiogram stimuli consisted of tone bursts ( $2 \mathrm{~ms} \pm 1 \mathrm{~ms}$ on/off ramps) of varying frequency and intensity.

\section{Monaural Auditory Brain Stem Responses}

Broadband click stimuli (i.e., $0.1 \mathrm{~ms}$ transient) were presented to each ear independently to generate monaural evoked potentials. Peak amplitude (i.e., the voltage from peak to trough) and latency (i.e., time to peak amplitude) were measured across the four peaks of the ABR waveform at $90 \mathrm{~dB}$ SPL (Figure 2A). The trough was considered the lowest point for that wave. Monaural data from the two ears were averaged to determine the monaural amplitude and latency for each animal. Similar to hearing thresholds across frequency, click threshold was determined for each genotype and sex. Click threshold is determined by decreasing the intensity of sound in 5-10 dB SPL steps until ABR waveforms disappear.

\section{Binaural Auditory Brain Stem Responses}

Broadband click stimuli at $90 \mathrm{~dB}$ SPL were also presented to both ears simultaneously to generate a binaural evoked potential. The BIC of the ABR was calculated by subtracting the sum of the two monaural ABRs from the binaural ABR (Laumen et al., 2016; Benichoux et al., 2018) (Figures 2B,C). BIC amplitude and latency were then measured using the custom Python software, with amplitude being relative to the zero baselines of the measurement (Figure 2C, gray area with line). BIC was characterized as the prominent negative DN1 wave corresponding to the fourth wave of the binaural and summed ABR (Figure 2B). To measure ITD computation using the BIC, animals were presented with stimuli that had varying ITDs of $\pm 2 \mathrm{~ms}$ in $0.5 \mathrm{~ms}$ steps, and corresponding BIC amplitudes and latencies were calculated like above. This ITD range was

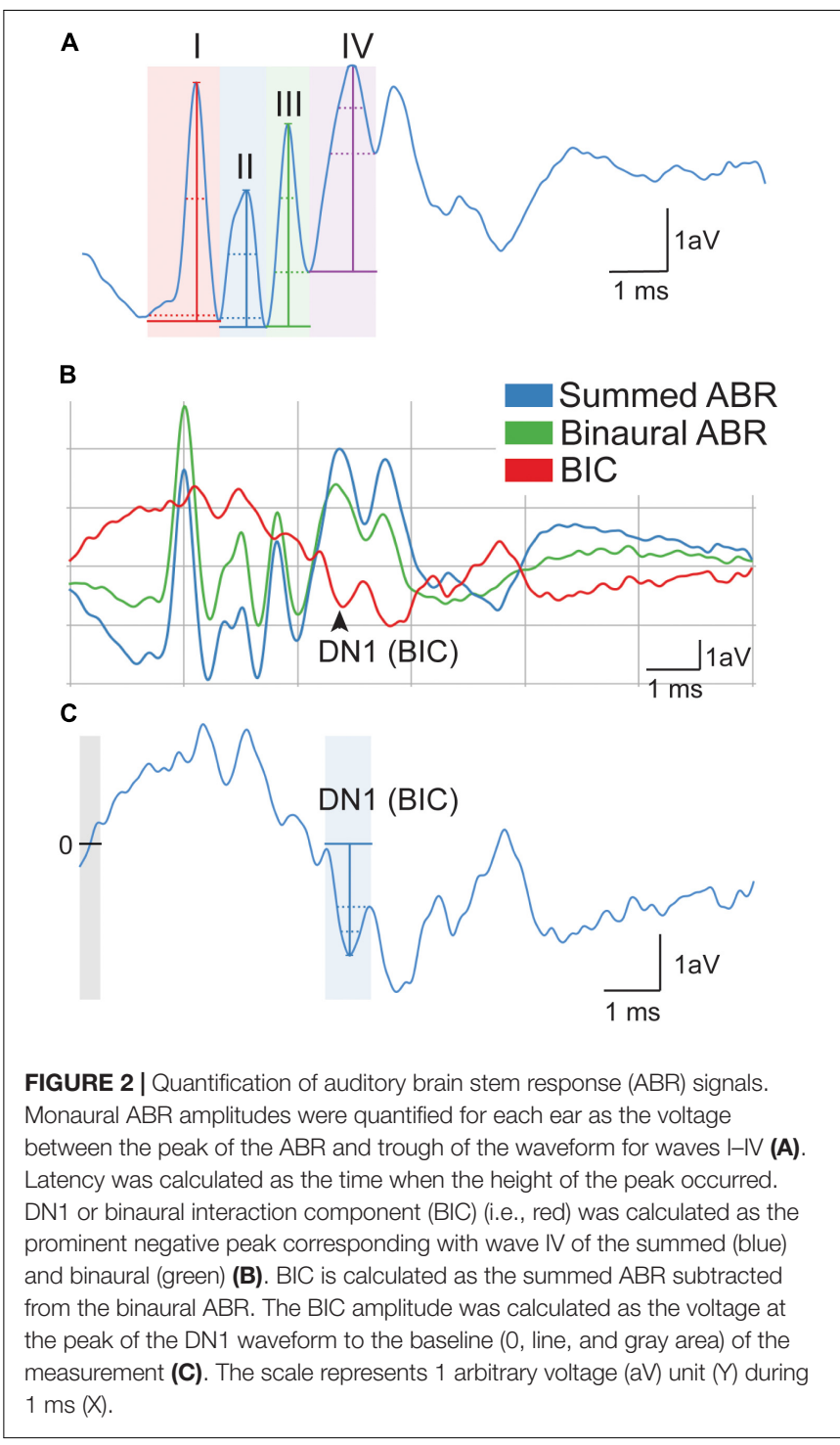

chosen to be comparable to other studies in small rodents (Benichoux et al., 2018).

\section{Analysis of Auditory Brain Stem Response Waveforms}

The custom python software was used to analyze evoked potentials for monaural and binaural stimuli (New et al., 2021). To account for fluctuation in the baseline signal of the ABR, raw traces were zeroed to establish a baseline across traces. The software included automatic peak detection with the capability of manual correction or deselection upon visual confirmation.

\section{Statistical Analyses}

Figures were generated using R Studio (R Core Team, 2013), ggplot2 (Wickham, 2016), and Adobe Illustrator (Adobe, San Jose, CA, United States) software. Data points in Figures 3, 4, and 5 represent means, error bars reflect standard error, boxplots in Figure 1 display the median and 25-75th percentiles (or 1st 

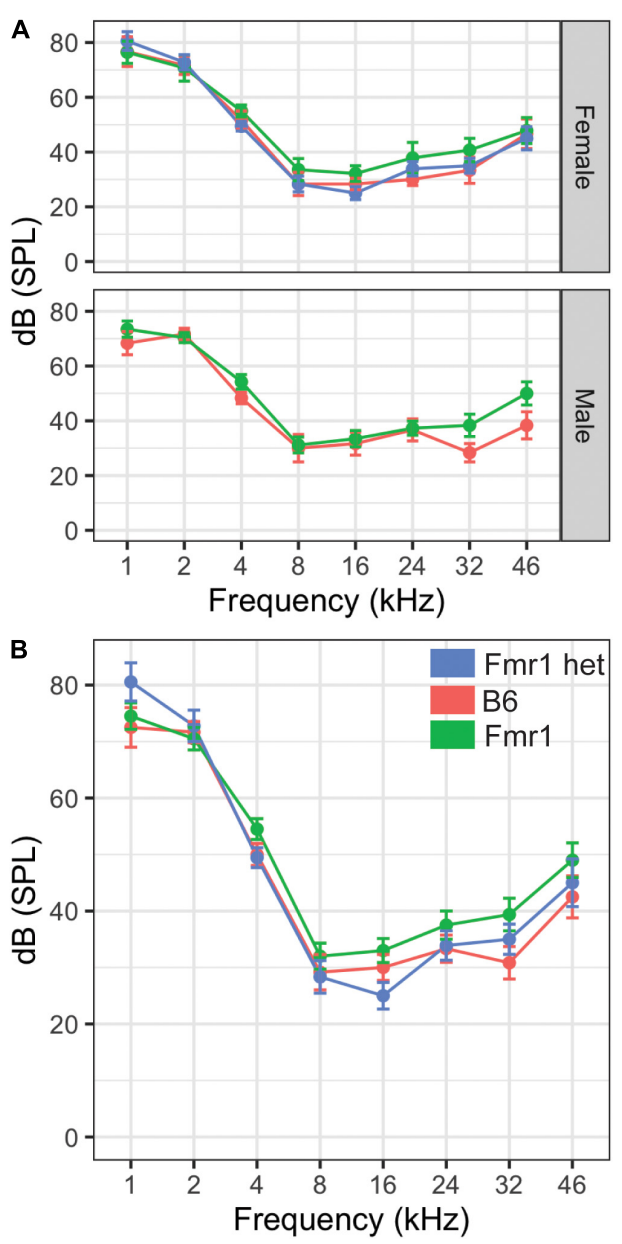

FIGURE 3 | Hearing threshold (dB SPL) was measured across frequencies $(1-46 \mathrm{kHz})$ in male and female mice of all genotypes (A). There were no differences in the hearing range between Fmr1 (green), B6 (red), and Fmr1 het (blue) mice of either sex (top panel A). When sexes were combined, there was no significant difference in hearing across frequencies (B). Data represent 6 B6, 7 Fmr1, 9 Fmr1 het females and 6 B6, 11 Fmr1 males.

and 3rd quartiles, respectively), the whiskers represent \pm 1.5 times the interquartile range. The data that falls outside the range are plotted as individual points. Multivariate data (i.e., monaural peak amplitude and latency, audiogram, and BIC amplitude and latency across ITD) were analyzed using linear mixed effects (lme4) models (Bates et al., 2015) with sex, genotype, litter, and condition (i.e., ITD, frequency, peak) as fixed effects and animal as a random effect. It was expected that there may be differences between the sexes and genotypes; therefore, a priori, it was determined that estimated marginal means [emmeans; (Lenth, 2019)] would be used for pairwise comparisons between sexes and genotype. Two-way ANOVAs were performed to compare relationships between morphological features, sex, and genotype with the adjusted Tukey post hoc analysis to compare groups. Where values are indicated as statistically significant between the two genotypes, $*$ indicated a $p$-value of $<0.05,{ }^{* *} p<0.01$, and $* * * p<0.0001$.

\section{RESULTS}

We used both morphological and physiological features to examine hearing differences in a commonly used mouse model with FXS, C57BL/6J across genotypes and sexes. Hearing measurements included the frequency hearing range, monaural hearing ability, and binaural processing using the ABR, while morphological features included pinna and head measurements.

\section{Morphological Features}

People with FXS have altered craniofacial features, including large ears (Loesch et al., 1988). Consistent with our previous work (McCullagh et al., 2020a), we saw no difference between B6, Fmr1, or Fmr1 het animals for pinna attributes (Figure 1C pinna width, Figure 1D pinna length, Figure 1B effective diameter). In addition, pinna characteristics were the same between the sexes independent of genotype $(p=0.175$ pinna width, $p=0.96$ pinna length, $p=0.267$ effective diameter Figures 1B-D). When genotypes were compared within the same sex, there were no differences in weight, but sexes were significantly different independent of genotype $(p=0.0023)$ with females weighing significantly less than males. Similar to the pinna morphology, there was no significant difference in either distance between pinna or distance from the nose to pinna between the genotypes or sexes (Figures 1E,F). These data suggest that mice do not share the same craniofacial changes, at least in the measurements described here, as people with FXS.

\section{Hearing Range}

Our previous study showed that Fmr1 mice have increased thresholds for high-frequency hearing compared with those in B6 at $16 \mathrm{kHz}$ (McCullagh et al., 2020a). However, that study was limited by measuring only three frequencies (i.e., 4, 8, and $16 \mathrm{kHz}$ ) and seven mice of each genotype (i.e., combined sexes). Mice hear much higher frequencies than humans (Radziwon et al., 2009); therefore, we wanted to measure whether this high-frequency hearing loss exists across the frequencies in which mice hear in Fmr1 mutants and with a more in-depth sexspecific analysis. Interestingly, there were no differences between genotypes across the frequencies tested (Figure 3). There were no significant differences in hearing range between the sexes. Best frequencies for both genotypes, as indicated by lower threshold, of mice were between $8-46 \mathrm{kHz}$ consistent with specialized high frequency hearing.

\section{Monaural Hearing}

Amplitude and latency of monaural ABRs correspond with the neural activity across the ascending auditory pathway, with each wave representing different brain areas involved in the auditory processing (Alvarado et al., 2012). Other studies have shown both latency and amplitude alterations in the FVB mouse strain of Fmr1 mutation (Kim et al., 2013; Rotschafer et al., 2015; El-Hassar et al., 2019). We measured ABR responses of Fmr1 mutants to monaural click stimuli compared with B6 mutant mice to determine if they have a similar ABR phenotype to the 

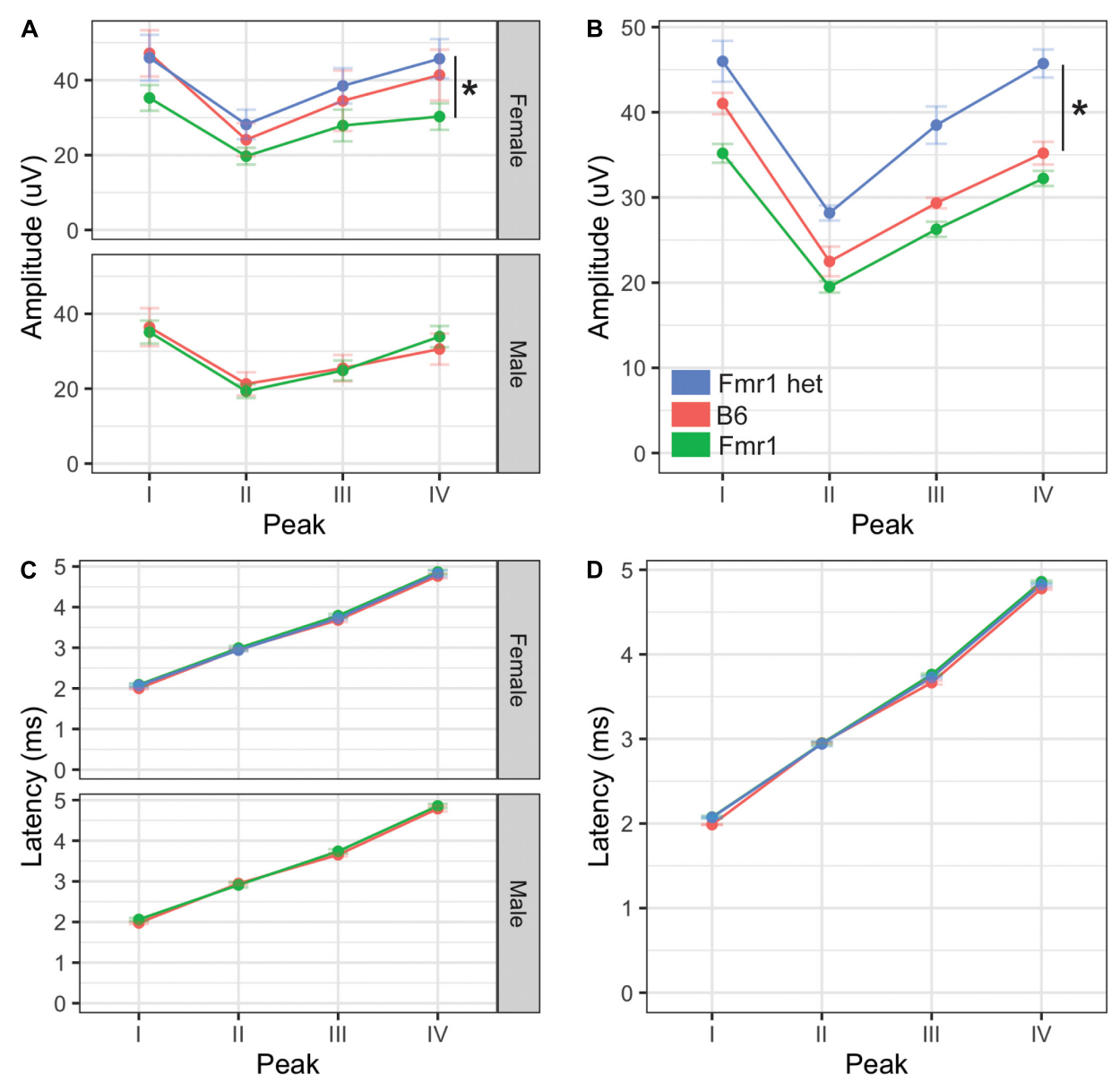

FIGURE 4 | Monaural hearing in mice with FXS. Monaural amplitudes and latencies for peaks I-IV of the ABR were recorded for Fmr1, Fmr1 het, and B6 animals. Peak IV amplitude was significantly lower in Fmr1 mice females compared with Fmr1 het females (A, upper). There were no significant differences in amplitudes for males (A, lower). When combined, there was a significant difference in Fmr1 het animals compared with Fmr1 (B). There was no difference in latency of peaks I-IV between sexes (C) or genotypes (D). ${ }^{*} p<0.05$. Data represent 6 B6, 12 Fmr1, and 9 Fmr1 het females and 8 B6 and 14 Fmr1 males.

FVB strain. We saw no differences in overall click threshold for either genotype or sex $(p=0.102$ genotype and $p=0.47$ for sex). The amplitude of monaural responses was significantly lower for wave IV of the ABR in Fmrl females compared with Fmr1 het females (Figure 4A upper). Indeed, Fmr1 het female amplitudes were closer to B6 than Fmr1 females, though Fmr1 females were not significantly different from B6. In contrast, Fmr1 male amplitudes for waves I-IV were not different from B6 (Figure 4A lower). When sexes were combined, Fmr1 het females had significantly higher amplitudes than B6 and were close to being significantly higher than Fmr1 mice $(p=0.0593)$. Consistent with sex driving the differences in genotype, peak amplitudes varied between the sexes. Female B6 mice had significantly higher amplitude peaks I and IV compared with B6 males $(p=0.0295$ peak I and $p=0.0289$ peak IV). In contrast, there were no sex differences between male and female Fmr1 mice, suggesting a more male-like phenotype (i.e., independent of genotype) in homozygous Fmr1 females. There were no differences between the sexes or genotypes in latency of monaural peaks (Figures 4C,D).

\section{Binaural Hearing}

While the monaural ABR provides information about binaural areas of the brain stem (i.e., potentially peaks III and IV), since they are elicited by either sound played directly to one ear (closed field) or equally to both ears (open field), little information can be gained about binaural integration of sound information. We used the BIC of the ABR to measure the ability of the binaural processing of the brain stem as the BIC varies with ITDs played to both ears. We saw no differences in amplitude of the BIC at any ITD between the two genotypes $(p=0.809)$ or with sex ( $p=0.6904$, Figures 5A,B), although there was a significant difference between Fmr1 male and female mouse BIC amplitudes at $1.5 \mathrm{~ms}$ ITD. There were no differences between the sexes for B6 mice for any ITD amplitude. Latency of the BIC was significantly slower in male Fmr1 compared with that in B6 males (Figure 5C, lower panel) only at 0 ITD, with no difference in genotype for female mice (Figure 5C, upper panel). When data were combined for sexes across genotypes, there was no significant difference in the latency of the BIC at any ITD (Figure 5D). There were differences in latency of the BIC between B6 (-1.5 ms) and Fmr1 

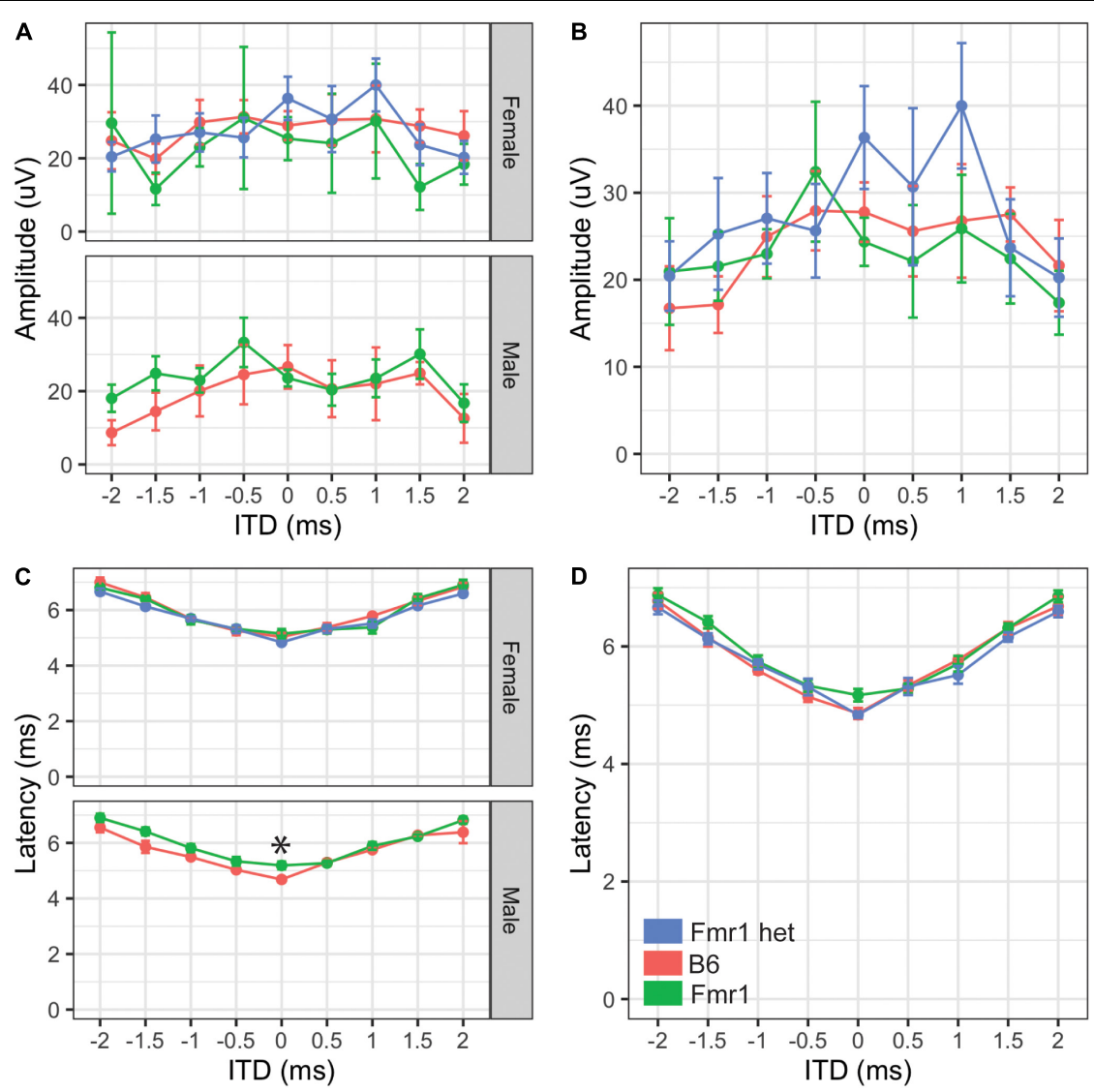

FIGURE 5 | Binaural hearing in Fragile X syndrome (FXS) mice. Binaural amplitudes and latencies for the BIC at ITDs between -2 to +2 ms in 0.5 ms steps were recorded for Fmr1 (green), Fmr1 het (blue), and B6 (red) animals. No differences in amplitude of the BIC with ITD for females (A, upper) or males (A, lower). When the sexes were combined, there was no significant difference in amplitude of the BIC with ITD (B). Fmr1 males had significantly longer latency of the BIC at 0 ITD compared with B6 males (C, lower), while there was no difference in latency of female responses (C, upper). When the sexes were combined, there was no difference in the BIC latency across ITDs between the genotypes (D). ${ }^{*} p<0.05$. Data represent 6 B6, 7 Fmr1, and 9 Fmr1 het females and 6 B6 and 9 Fmr1 males.

(1 ms) males and females although there was no overall main effect of $\operatorname{sex}(p=0.3367)$.

\section{DISCUSSION}

This is the first study to characterize the ABR in the C57BL/6J Fmr1 mutant mouse and, in particular, highlights morphological characteristics, hearing range, monaural ABRs, and binaural integration across sexes and in heterozygote females. Consistent with previous study, we saw an increase in the hearing threshold at high frequencies in Fmrl mice, although this phenotype is male specific and no change in morphology (pinna or facial characteristics) (McCullagh et al., 2020a). Female Fmr1 mice have reduced wave IV amplitudes of the monaural ABR, and wild-type females have increased wave I and IV amplitudes compared with B6 males, suggesting that female Fmr1 mice have a more malelike phenotype for monaural ABR amplitude. Finally, we showed that male Fmr1 mice have increased latency of the BIC at 0 ITD but not other ITDs or changes in amplitude of the BIC across ITD compared with B6 animals, suggesting changes in the timing of the processing of binaural information that does not change overall ITD following ability.
The pinnae size and shape are the first two features available to determine sound localization ability in animals with external ears (Butler, 1975; Musicant and Butler, 1984). Craniofacial alterations including prominent ears and elongated face are hallmark features of humans with FXS (Loesch et al., 1988; Heulens et al., 2013) and indeed may be a factor in auditory hypersensitivity that has been underexplored. Consistent with our previous study, we saw no alterations in the pinna or facial characteristics in the C57BL/6J mouse model with FXS (McCullagh et al., 2020a) using calipers as a measurement tool. Others have explored differences in the morphological skull in mice with FXS using different tools, such as CT/MRI (Ellegood et al., 2010) and micro-CT (Heulens et al., 2013) with mixed results. Heulens et al., 2013 showed alterations in skull and jaw characteristics that had not been characterized previously with a similar technique (Ellegood et al., 2010) although differences may be due to how features were measured. We also saw no difference in weight of Fmr1 animals compared with the wildtype, which is in contrast to our previous study where we noted that Fmr1 animals weighed less than wild-type (McCullagh et al., 2020a) and others that showed an increase in male Fmr1 mouse weight compared with the wild-type (Leboucher et al., 2019). Differences in weight may be due to the inclusion of female 
animals (McCullagh et al., 2020a) and older animals (Leboucher et al., 2019). Overall changes in pinna morphology may still be an important factor in sound localization ability in Fmr1 animals and should be explored with more detailed techniques to determine if increased pinna measures in both humans and animal models may underly some aspect of auditory hypersensitivity symptomology.

Our previous results showed increased hearing thresholds at high frequencies $(16 \mathrm{kHz})$ measured by ABR in the C57BL/6J Fmrl strain with data combined for the sexes (McCullagh et al., 2020a). In the current study, we do not see increased thresholds at $16 \mathrm{kHz}$ but do see a trend towards increased thresholds at higher frequencies in male Fmrl mice specifically, though not significant. These data are consistent with the increased thresholds across frequencies seen in adult male FVB Fmr1 mice (Rotschafer et al., 2015), though note that there was no change in threshold across frequencies in males of the same FVB strain at younger ages (Kim et al., 2013; El-Hassar et al., 2019). Additional studies should examine the hearing range across development and sexes in both strains to further show whether loss of highfrequency hearing is a conserved feature in FXS.

Previous studies in the FVB Fmr1 mouse line show a robust wave I amplitude decrease in males across ages (Rotschafer et al., 2015; El-Hassar et al., 2019), although see Kim et al. (2013). We did not see any change in wave I amplitude in the C57BL/6J Fmrl line in adult animals of either sex. These conflicting results may be in part due to the earlier onset age-related hearing loss, which can be seen as decreases in early waves of the ABR, that occurs in the B6 background (Hunter and Willott, 1987). Changes in wave I amplitude specific to FXS may be masked by overall decreases in wave I amplitude across genotypes in this background. Interestingly, data in male FVB Fmrl mice show no differences (adults, Kim et al., 2013; Rotschafer et al., 2015) or increased amplitudes in wave IV of the ABR (young, ElHassar et al., 2019), whereas our data show a decreased wave IV amplitude in Fmrl females on the B6 background. These differences again may be due to differences in sexes and ages of animals tested. Finally, our finding of no difference in latency of monaural waves is consistent with the majority of the work in FVB mice (Rotschafer et al., 2015; El-Hassar et al., 2019), although note that Kim et al., 2013 showed shorter latency for wave I. Our data further add to the knowledge of ABR phenotypes that might be consistent across genotypes.

While ours is the first study to characterize the BIC in an FXSmutant mouse strain, our data are consistent with the BIC as it varies with ITD in mice (Benichoux et al., 2018). Namely, mice have a small range of ITD cues available due to their small head size, and therefore, the BIC amplitude decreases with increasing ITD between the ears, but this overall amplitude change is smaller than animals with more dominant ITD hearing ability (such as chinchilla or cats)(Benichoux et al., 2018). Additionally, consistent with previous study, the BIC latency gets longer with increasing ITD (Ferber et al., 2016; Laumen et al., 2016; Benichoux et al., 2018). Interestingly, our work in mice with FXS is consistent with an increased latency of the BIC seen in a study in autistic people (ElMoazen et al., 2019), although they also see a decrease in the amplitude of the BIC. Our findings that the BIC latency is only significant in males at 0 ITD potentially suggest that there is overall slowing of binaural processing in the brain stem, which may ultimately impact binaural hearing, but that it is not dependent on ITD, which would be consistent with mice that do not rely as predominantly on ITD cues compared with other species. In addition, while these results do not directly measure auditory hypersensitivity, underlying alterations to the timing of brain stem or amplitude of brain stem regions will impact later processing of this information as it moves through the ascending auditory pathway to other subcortical and cortical areas.

The subject of sex differences in animal models is important for fully understanding the complexities of disorders such as ASD or FXS, which seem to impact females differently than males (Werling and Geschwind, 2013; Nolan et al., 2017). In FXS, due to it being an X-linked disorder, there is a higher prevalence in males than females, which can undergo X-inactivation on the effected $\mathrm{X}$ chromosome (i.e., genetic mosaicism) (Kirchgessner et al., 1995). However, mice offer a unique opportunity to measure both heterozygote and homozygous females giving insight into potential sex differences related to loss of Fmr1 on one or both X chromosomes. Our data suggest that there are indeed differences in auditory phenotypes between heterozygous and homozygous females (wave IV amplitude) in addition to differences between males and females. These and future data comparing female Fmrl subtypes may give insight into the role of X-inactivation in phenotypes of auditory brain stem processing.

\section{CONCLUSION}

This study offers important insight into auditory phenotypes that may be shared or differ between background strains of mice with FXS. In addition, while subtle, we showed sex-specific and full or heterozygote mutation-specific differences in the auditory brain stem function for both monaural and binaural hearing in B6 background mice. Further studies measuring auditory phenotypes for B6 mice in earlier ages across the sexes would be useful to further characterize potential similarities compared with the FVB Fmr1 strain. In addition, characterizing the BIC in the FVB strain would be useful to elucidate if latency phenotypes are consistent across backgrounds.

\section{DATA AVAILABILITY STATEMENT}

The raw data supporting the conclusions of this article will be made available by the authors, without undue reservation.

\section{ETHICS STATEMENT}

The animal study was reviewed and approved by Oklahoma State University IACUC.

\section{AUTHOR CONTRIBUTIONS}

EM and AC collected the data for the manuscript. EM performed the statistical analyses, created the figures for the manuscript, and 
developed the ideas and methods. Both authors helped write and revise the manuscript.

\section{FUNDING}

Supported by NIH 1R15HD105231-01. Preliminary work was also funded by a FRAXA research grant and $\mathrm{NIH}$ 3T32DC012280-05S1.

\section{REFERENCES}

Abbeduto, L., and Hagerman, R. J. (1997). Language and communication in fragile X syndrome. Ment. Retard. Dev. Disabil. Res. Rev. 3, 313-322. doi: 10.1002/ (SICI) 1098-277919973:4<313::AID-MRDD6<3.0.CO;2-O

Alvarado, J. C., Fuentes-Santamaría, V., Jareño-Flores, T., Blanco, J. L., and Juiz, J. M. (2012). Normal variations in the morphology of auditory brainstem response (ABR) waveforms: a study in Wistar rats. Neurosci. Res. 73, 302-311. doi: 10.1016/j.neures.2012.05.001

Anbuhl, K. L., Benichoux, V., Greene, N. T., Brown, A. D., and Tollin, D. J. (2017). Development of the head, pinnae, and acoustical cues to sound location in a precocial species, the guinea pig (Cavia porcellus). Hear Res. 356, 35-50. doi: 10.1016/j.heares.2017.10.015

Arnett, M. T., Herman, D. H., and McGee, A. W. (2014). Deficits in tactile learning in a mouse model of fragile X syndrome. PLoS One 9:e109116. doi: 10.1371/ journal.pone.0109116

Bates, D., Mächler, M., Bolker, B., and Walker, S. (2015). Fitting linear mixedeffects models using lme4. J. Statist. Softw. 67, 1-48. doi: 10.18637/jss.v0 67.i01

Beebe, K., Wang, Y., and Kulesza, R. (2014). Distribution of fragile X mental retardation protein in the human auditory brainstem. Neuroscience 273, 79-91. doi: 10.1016/j.neuroscience.2014.05.006

Benichoux, V., Ferber, A., Hunt, S., Hughes, E., and Tollin, D. (2018). Across species "natural ablation" reveals the brainstem source of a noninvasive biomarker of binaural hearing. J. Neurosci. 38, 8563-8573. doi: 10.1523/ JNEUROSCI.1211-18.2018

Beutelmann, R., Laumen, G., Tollin, D., and Klump, G. M. (2015). Amplitude and phase equalization of stimuli for click evoked auditory brainstem responses. J. Acoust. Soc. Am. 137, EL71-EL77. doi: 10.1121/1.4903921

Brittan-Powell, E. F., and Dooling, R. J. (2004). Development of auditory sensitivity in budgerigars (Melopsittacus undulatus). J. Acoust. Soc. Am. 115, 3092-3102. doi: $10.1121 / 1.1739479$

Bronkhorst, A. W. (2015). The cocktail-party problem revisited: early processing and selection of multi-talker speech. Atten. Percept. Psychophys. 77, 1465-1487. doi: 10.3758/s13414-015-0882-9

Brown, M. R., Kronengold, J., Gazula, V.-R., Chen, Y., Strumbos, J. G., Sigworth, F. J., et al. (2010). Fragile $\mathrm{X}$ mental retardation protein controls gating of the sodium-activated potassium channel Slack. Nat. Neurosci. 13, 819-821. doi: $10.1038 / \mathrm{nn} .2563$

Butler, R. A. (1975). "The influence of the external and middle ear on auditory discriminations," in Auditory System: Physiology (CNS) Behavioral Studies Psychoacoustics Handbook of Sensory Physiology, eds M. Abeles, R. A. Bredberg, J. Butler, H. Casseday, J. E. Desmedt, I. T. Diamond, et al. (Berlin: Springer), 247-260. doi: 10.1007/978-3-642-65995-9_6

Chen, L., and Toth, M. (2001). Fragile X mice develop sensory hyperreactivity to auditory stimuli. Neuroscience 103, 1043-1050. doi: 10.1016/s0306-4522(01) 00036- 7

Curry, R. J., Peng, K., and Lu, Y. (2018). Neurotransmitter- and release-modespecific modulation of inhibitory transmission by group I metabotropic glutamate receptors in central auditory neurons of the mouse. J. Neurosci. 38, 8187-8199. doi: 10.1523/JNEUROSCI.0603-18.2018

Dahlhaus, R. (2018). Of men and mice: modeling the fragile X syndrome. Front. Mol. Neurosci. 11:41. doi: 10.3389/fnmol.2018.00041

El-Hassar, L., Song, L., Tan, W. J. T., Large, C. H., Alvaro, G., Santos-Sacchi, J., et al. (2019). Modulators of Kv3 potassium channels rescue the auditory function of

\section{ACKNOWLEDGMENTS}

We would like to thank members of the McCullagh lab and team mouse, including Ishani Ray and Sabiha Alam, which assisted with ABRs. Furthermore, we would like to acknowledge Shani Poleg and Daniel Tollin for helping us set up these experiments in Colorado and continue them in Oklahoma.

fragile X mice. J. Neurosci. 39, 4797-4813. doi: 10.1523/JNEUROSCI.0839-18. 2019

Ellegood, J., Pacey, L. K., Hampson, D. R., Lerch, J. P., and Henkelman, R. M. (2010). Anatomical phenotyping in a mouse model of fragile $\mathrm{X}$ syndrome with magnetic resonance imaging. Neuroimage 53, 1023-1029. doi: 10.1016/j. neuroimage.2010.03.038

ElMoazen, D., Sobhy, O., Abdou, R., and Ismail, H. (2019). Binaural interaction component of the auditory brainstem response in children with autism spectrum disorder. Int. J. Pediat. Otorhinolaryngol. 19:109850. doi: 10.1016/j. ijporl.2019.109850

Ethridge, L. E., White, S. P., Mosconi, M. W., Wang, J., Pedapati, E. V., Erickson, C. A., et al. (2017). Neural synchronization deficits linked to cortical hyperexcitability and auditory hypersensitivity in fragile X syndrome. Mol. Autism 8:140. doi: 10.1186/s13229-017-0140-1

Ferber, A. T., Benichoux, V., and Tollin, D. J. (2016). Test-retest reliability of the binaural interaction component of the auditory brainstem response. Ear Hear 37, e291-e301. doi: 10.1097/AUD.0000000000000315

Garcia-Pino, E., Gessele, N., and Koch, U. (2017). Enhanced excitatory connectivity and disturbed sound processing in the auditory brainstem of fragile $\mathrm{X}$ mice. J. Neurosci. 37, 7403-7419. doi: 10.1523/JNEUROSCI.2310-16.2017

Grothe, B., Pecka, M., and McAlpine, D. (2010). Mechanisms of sound localization in mammals. Physiol. Rev. 90, 983-1012. doi: 10.1152/physrev.00026.2009

Hagerman, R. J., and Hagerman, P. J. (2002). Fragile X Syndrome: Diagnosis, Treatment and Research. Baltimore, MY: Johns Hopkins University Press.

Heulens, I., Suttie, M., Postnov, A., De Clerck, N., Perrotta, C. S., Mattina, T., et al. (2013). Craniofacial characteristics of fragile X syndrome in mouse and man. Eur. J. Hum. Genet. 21, 816-823. doi: 10.1038/ejhg.2012.265

Hunter, K. P., and Willott, J. F. (1987). Aging and the auditory brainstem response in mice with severe or minimal presbycusis. Hear Res. 30, 207-218. doi: 10.1016/ 0378-5955(87)90137-7

Kim, H., Gibboni, R., Kirkhart, C., and Bao, S. (2013). Impaired critical period plasticity in primary auditory cortex of fragile X model mice. J. Neurosci. 33, 15686-15692. doi: 10.1523/JNEUROSCI.3246-12.2013

Kirchgessner, C. U., Warren, S. T., and Willard, H. F. (1995). X inactivation of the FMR1 fragile X mental retardation gene. J. Med. Genet. 32, 925-929. doi: 10.1136/jmg.32.12.925

Laumen, G., Ferber, A. T., Klump, G. M., and Tollin, D. J. (2016). The physiological basis and clinical use of the binaural interaction component of the auditory brainstem response. Ear Hear 37, e276-e290. doi: 10.1097/AUD. 0000000000000301

Leboucher, A., Bermudez-Martin, P., Mouska, X., Amri, E.-Z., Pisani, D. F., and Davidovic, L. (2019). Fmr1-deficiency impacts body composition, skeleton, and bone microstructure in a mouse model of fragile X syndrome. Front. Endocrinol. (Lausanne) 10:678. doi: 10.3389/fendo.2019.00678

Lenth, R. (2019). emmeans: Estimated Marginal Means, aka Least-Squares Means. Available online at: https://CRAN.R-project.org/package=emmeans

Levine, R. A. (1981). Binaural interaction in brainstem potentials of human subjects. Ann. Neurol. 9, 384-393. doi: 10.1002/ana.410090412

Loesch, D. Z., Lafranchi, M., and Scott, D. (1988). Anthropometry in martin-bell syndrome. Am. J. Med. Genet. 30, 149-164. doi: 10.1002/ajmg.1320300113

Lovelace, J. W., Ethell, I. M., Binder, D. K., and Razak, K. A. (2018). Translationrelevant EEG phenotypes in a mouse model of Fragile X Syndrome. Neurobiol. Dis. 115, 39-48. doi: 10.1016/j.nbd.2018.03.012

Lovelace, J. W., Rais, M., Palacios, A. R., Shuai, X., Bishay, S., Popa, O., et al. (2020). Deletion of Fmr1 from forebrain excitatory neurons triggers abnormal cellular, 
EEG and behavioral phenotypes in the auditory cortex of a mouse model of Fragile X Syndrome. Cereb. Cortex 30, 969-988. doi: 10.1093/cercor/bhz141

$\mathrm{Lu}, \mathrm{Y}$. (2019). Subtle differences in synaptic transmission in medial nucleus of trapezoid body neurons between wild-type and Fmr1 knockout mice. Brain Res. 1717, 95-103. doi: 10.1016/j.brainres.2019.04.006

McCullagh, E. A., Poleg, S., Greene, N. T., Huntsman, M. M., Tollin, D. J., and Klug, A. (2020a). Characterization of auditory and binaural spatial hearing in a fragile X syndrome mouse model. eNeuro 7:ENEURO.0300-19.2019. doi: 10.1523/ENEURO.0300-19.2019

McCullagh, E. A., Rotschafer, S. E., Auerbach, B. D., Klug, A., Kaczmarek, L. K., Cramer, K. S., et al. (2020b). Mechanisms underlying auditory processing deficits in fragile X syndrome. FASEB J. 34, 3501-3518. doi: 10.1096/fj. 201902435R

McCullagh, E. A., Salcedo, E., Huntsman, M. M., and Klug, A. (2017). Tonotopic alterations in inhibitory input to the medial nucleus of the trapezoid body in a mouse model of Fragile X syndrome. J. Comparat. Neurol. 262:375. doi: $10.1002 /$ cne. 24290

Musicant, A. D., and Butler, R. A. (1984). The influence of pinnae-based spectral cues on sound localization. J. Acoust. Soc. Am. 75, 1195-1200. doi: 10.1121/1. 390770

New, E. M., Li, B.-Z., Lei, T., and McCullagh, E. A. (2021). Hearing Ability of Prairie Voles (Microtus ochrogaster). **. doi: 10.1101/2021.10.07.463519

Nolan, S. O., Reynolds, C. D., Smith, G. D., Holley, A. J., Escobar, B., Chandler, M. A., et al. (2017). Deletion of Fmr1 results in sex-specific changes in behavior. Brain Behavior 7:e00800. doi: 10.1002/brb3.800

R Core Team (2013). R: A Language and Environment for Statistical Computing. Vienna: R Foundation for Statistical Computing.

Radziwon, K. E., June, K. M., Stolzberg, D. J., Xu-Friedman, M. A., Salvi, R. J., and Dent, M. L. (2009). Behaviorally measured audiograms and gap detection thresholds in CBA/CaJ mice. J. Comp. Physiol. A Neuroethol. Sens. Neural Behav. Physiol. 195, 961-969. doi: 10.1007/s00359-009-0472-1

Rais, M., Binder, D. K., Razak, K. A., and Ethell, I. M. (2018). Sensory processing phenotypes in fragile X syndrome. ASN Neuro 10, 1-19. doi: 10.1177/ 1759091418801092

Razak, K. A., Binder, D. K., and Ethell, I. M. (2021). Neural correlates of auditory hypersensitivity in fragile X syndrome. Front. Psychiatry 12:1544. doi: 10.3389/ fpsyt.2021.720752

Rotschafer, S. E., and Cramer, K. S. (2017). Developmental emergence of phenotypes in the auditory brainstem nuclei of Fmr1Knockout mice. eNeuro 4, 1-21. doi: 10.1523/ENEURO.0264-17.2017

Rotschafer, S. E., Marshak, S., and Cramer, K. S. (2015). Deletion of Fmr1 alters function and synaptic inputs in the auditory brainstem. PLoS One 10:e0117266. doi: 10.1371/journal.pone.0117266

Santos, M., Marques, C., Nóbrega Pinto, A., Fernandes, R., Coutinho, M. B., Almeida, E., et al. (2017). Autism spectrum disorders and the amplitude of auditory brainstem response wave I. Autism. Res. 10, 1300-1305. doi: 10.1002/ aur. 1771

Sinclair, D., Oranje, B., Razak, K. A., Siegel, S. J., and Schmid, S. (2017). Sensory processing in autism spectrum disorders and Fragile $\mathrm{X}$ syndrome-From the clinic to animal models. Neurosci. Biobehav. Rev. 76, 235-253. doi: 10.1016/j. neubiorev.2016.05.029

Stefanelli, A. C. G. F., Zanchetta, S., Furtado, E. F., Stefanelli, A. C. G. F., Zanchetta, S., and Furtado, E. F. (2020). Auditory hyper-responsiveness in autism spectrum disorder, terminologies and physiological mechanisms involved: systematic review. CoDAS 32:287. doi: 10.1590/2317-1782/20192018287

The Dutch-Belgian Fragile X Consorthium, Bakker, C. E., Verheij, C., Willemsen, R., van der Helm, R., Oerlemans, F., et al. (1994). Fmr1 knockout mice: a model to study fragile X mental retardation. Cell 78, 23-33. doi: 10.1016/00928674(94)90569-X

Tian, Y., Yang, C., Shang, S., Cai, Y., Deng, X., Zhang, J., et al. (2017). Loss of FMRP impaired hippocampal long-term plasticity and spatial learning in rats. Front. Mol. Neurosci. 10:269. doi: 10.3389/fnmol.2017. 00269

Till, S. M., Asiminas, A., Jackson, A. D., Katsanevaki, D., Barnes, S. A., Osterweil, E. K., et al. (2015). Conserved hippocampal cellular pathophysiology but distinct behavioural deficits in a new rat model of FXS. Hum. Mol. Genet. 24, 5977-5984. doi: 10.1093/hmg/ddv299

Visser, E., Zwiers, M. P., Kan, C. C., Hoekstra, L., van Opstal, A. J., and Buitelaar, J. K. (2013). Atypical vertical sound localization and sound-onset sensitivity in people with autism spectrum disorders. J. Psychiatry Neurosci. 38, 398-406. doi: 10.1503/jpn.120177

Wang, T., de Kok, L., Willemsen, R., Elgersma, Y., and Borst, J. G. G. (2015). In vivo synaptic transmission and morphology in mouse models of Tuberous sclerosis, Fragile X syndrome, Neurofibromatosis type 1, and Costello syndrome. Front. Cell Neurosci. 9:234. doi: 10.3389/fncel.2015.00234

Wang, Y., Sakano, H., Beebe, K., Brown, M. R., de Laat, R., Bothwell, M., et al. (2014). Intense and specialized dendritic localization of the fragile $\mathrm{X}$ mental retardation protein in binaural brainstem neurons: a comparative study in the alligator, chicken, gerbil, and human: FMRP localization in NL/MSO dendrites. J. Comparat. Neurol. 522, 2107-2128. doi: 10.1002/cne.23 520

Werling, D. M., and Geschwind, D. H. (2013). Sex differences in autism spectrum disorders. Curr. Opin. Neurol. 26, 146-153. doi: 10.1097/WCO. 0b013e32835ee548

Wickham, H. (2016). ggplot2: Elegant Graphics for Data Analysis. New York, NY: Springer-Verlag.

Conflict of Interest: The authors declare that the research was conducted in the absence of any commercial or financial relationships that could be construed as a potential conflict of interest.

The handling editor declared a past co-authorship with one of the authors EM.

Publisher's Note: All claims expressed in this article are solely those of the authors and do not necessarily represent those of their affiliated organizations, or those of the publisher, the editors and the reviewers. Any product that may be evaluated in this article, or claim that may be made by its manufacturer, is not guaranteed or endorsed by the publisher.

Copyright $\odot 2022$ Chawla and McCullagh. This is an open-access article distributed under the terms of the Creative Commons Attribution License (CC BY). The use, distribution or reproduction in other forums is permitted, provided the original author(s) and the copyright owner(s) are credited and that the original publication in this journal is cited, in accordance with accepted academic practice. No use, distribution or reproduction is permitted which does not comply with these terms. 